\title{
Controlled electrospray generation of non-spherical alginate microparticles
}

\author{
Morteza Jeyhani, ${ }^{[a, b, c]}$ Sze Yi Mak, ${ }^{[d, ~ e] ~ S t e p h e n ~ S a m m u t, ~}{ }^{[b, c, ~ f]}$ Ho Cheung Shum, ${ }^{[d, ~ e] ~ D a e ~ K u n ~}$ \\ Hwang ${ }^{*[b, c, g]}$ and Scott S. H. Tsai ${ }^{*[a, b, c]}$
}

\begin{abstract}
Electrospraying is a technique used to generate microparticles in a high throughput manner. For biomedical applications, a biocompatible electrosprayed material is often desirable. Using polymers, such as alginate hydrogels, makes it possible to create biocompatible and biodegradable microparticles that can be used for cell encapsulation, to be employed as drug carriers, and for use in $3 \mathrm{D}$ cell culturing. Evidence in the literature suggests that the morphology of the biocompatible microparticles is relevant in controlling the dynamics of the microparticles in drug delivery and 3D cell culturing applications. Yet, most electrospray-based techniques only form spherical microparticles, and there is currently no widely adopted technique for producing non-spherical microparticles at a high throughput. Here, we demonstrate the generation of non-spherical biocompatible alginate microparticles by electrospraying, and control the shape of the microparticles by varying experimental parameters such as chemical concentration and the distance between the electrospray tip and the particle-solidification bath. Importantly, we show that these changes to the experimental setup enable the synthesis of different shaped particles, and the systematic change in parameters, such as chemical concentration, result in monotonic changes to the particle aspect ratio. We expect that these results will find utility in many biomedical applications that require biocompatible microparticles of specific shapes.
\end{abstract}

Keywords: non-spherical microparticles $•$ drug delivery $\bullet$ electrospray technique $\bullet$ high throughput

[a] Department of Mechanical and Industrial Engineering Ryerson University

350 Victoria St., Toronto, ON M5B 2K3

Canada

E-mail:scott.tsai@ryerson.ca

[b] M. Jeyhani, S. Sammut, Dr. S. S. H. Tsai, Dr. D. K. Hwang

Keenan Research Centre for Biomedical Science

St. Michael's Hospital

30 Bond St., Toronto, ON M5B 1W8

Canada

[c] M. Jeyhani, S. Sammut, Dr. S. S. H. Tsai, Dr. D. K. Hwang

Institute for Biomedical Engineering, Science, and Technology (iBEST)

a partnership between Ryerson University and St. Michael's Hospital

209 Victoria St., Toronto, ON M5B 1T8

Canada

[d] S. Y. Mak, Dr. H. C. Shum

Shenzhen Institute of Research and Innovation

Shenzhen, Guangdong, People's Republic of China

[e] S. Y. Mak, Dr. H. C. Shum

Department of Mechanical Engineering

University of Hong Kong

Pokfulam, Hong Kong

[f] S. Sammut

Department of Electrical and Computer Engineering

Ryerson University

350 Victoria St., Toronto, ON M5B 2K3

Canada

[g] Dr. D. K. Hwang

Department of Chemical Engineering

Ryerson University

350 Victoria St., Toronto, ON M5B 2K3

Canada

E-mail: dkhwang@ryerson.ca

Supporting information for this article is given via a link at the end of the document 


\section{Introduction}

Microparticles have found applications in a variety of different fields, including applications in pharmaceutical, food, pesticides, cosmetic industries, as well as biotechnological applications, for instance cell encapsulation for three-dimensional cell culturing for tissue engineering purposes. ${ }^{[1-4]}$ Specifically, biodegradable and biocompatible microparticles are useful for drug delivery applications because of their unique features such as their small size, drug encapsulation ability, bioavailability, reduced toxicity, and minimized adverse health effects in comparison to conventional drug delivery methods. ${ }^{[5]}$ These polymer drug carriers are often made with various biodegradable polymers, ${ }^{[6]}$ such as alginate. As a polysaccharide, alginate is one of the most used materials in applications such as the encapsulation of drugs, proteins, insulin, and cells in particles, ${ }^{[7-9]}$ in regenerative medicine, ${ }^{[10]} 3 \mathrm{D}$ culture, ${ }^{[11-14]}$ and tissue engineering. ${ }^{[15]}$

The vast majority of research on drug delivery particles only use particles that are spherical. ${ }^{[9],[16-18]}$ However, recent evidence in the literature suggests the emergence of the morphology of drug carrying particles as an important parameter controlling the mechanism of drug delivery. ${ }^{[5,8]}$ Namely, the shape of non-spherical particles affects the movement of particles in the presence of flow, ${ }^{[5]}$ and particle morphology impacts the drug release's pharmacokinetics, ${ }^{[19]}$ the mechanics of the drug carrier's transportation and circulation inside the body, ${ }^{[20,21]}$ and the targeting ability of the particles as drug delivery vehicles. ${ }^{[5]}$

Despite the fact that the morphology of alginate-based particles significantly affects the drug delivery mechanism, there are only a handful of manufacturing methods developed to tune the shape of alginate microparticles. Also, for gravity-driven droplet impact into calcium chloride baths, it is known that the impact energy, fluid viscosity, and surface tension all contribute to determine the final size and the shape of fabricated particles. ${ }^{[22-24]}$ The generation of non-spherical microparticles is possible using synthetic techniques such as lithography, ${ }^{[25-28]}$ photopolymerization, ${ }^{[25-27,29]}$ and microfluidics. ${ }^{[25-27]}$ However, barriers to generating shape-controllable alginate microparticles still persist. For example, combining microfluidics and photopolymerization is a well-developed technique to make highly tunable non-spherical particles. ${ }^{25-}$ 27,29-31] However, it is difficult to apply this method to generate non-spherical alginate microparticles because alginates need to be modified to be photocrosslinkable. ${ }^{[32]}$ Other methods that make non-spherical microparticles are challenged by low throughput (for example, $O(1-100)$ particles per second), ${ }^{[33,34]}$ and some cannot create microparticles that are less than hundreds of microns in diameter. ${ }^{[24]}$

Here, we describe a new method, based on electrospraying, to manufacture non-spherical alginate microparticles. Electrosprays are able to generate microparticles at rates up to $4 \times 10^{5}$ particles per second, ${ }^{[35]}$ so this new method should overcome the low-throughput challenges associated with current techniques used to make non-spherical microparticles. ${ }^{[34]}$ Our technique achieves alginate microparticles with tunable shapes, by adjusting several electrospray experimental parameters. Namely, we adjust the chemical initiator concentration of the liquid bath, and the distance from the metallic ring of the electrospray system to the free-surface of the liquid bath below. The liquid-air surface tension and liquid viscosities also play a role in controlling the resulting particle shape. ${ }^{[23]}$ However, these variables are kept constant in our experiments (The values of these variables are provided in Tables S1 and S2 in Supporting Information). 
In this manuscript, we first report our experimental observation of the different alginate microparticle morphologies achievable with our electrospray setup. We build a phase diagram of the possible particle shapes by systematically changing two experimental parameters: the chemical initiator concentration in the liquid bath and the distance from the metallic ring to the free surface of the bath. We also show that, for hemispherical shape microparticles, the particle aspect ratio-the particle height divided by its base-is controllable by the two experimental parameters. We further demonstrate that the action of stirring the liquid bath enables an additional interesting morphology-microparticles that have tail-like features whose curvature changes with the liquid stir rate. Finally, we demonstrate that the technique can be applied to make magnetizable non-spherical particles, and cell encapsulating non-spherical microcapsules. We anticipate that this approach to make non-spherical alginate microparticles will be able to address biomedical needs, including improving the efficacy of drug delivery, and enabling cell encapsulation and $3 \mathrm{D}$ cell culturing.

\section{Results and Discussion}

\subsection{Controlling the particles' morphology}

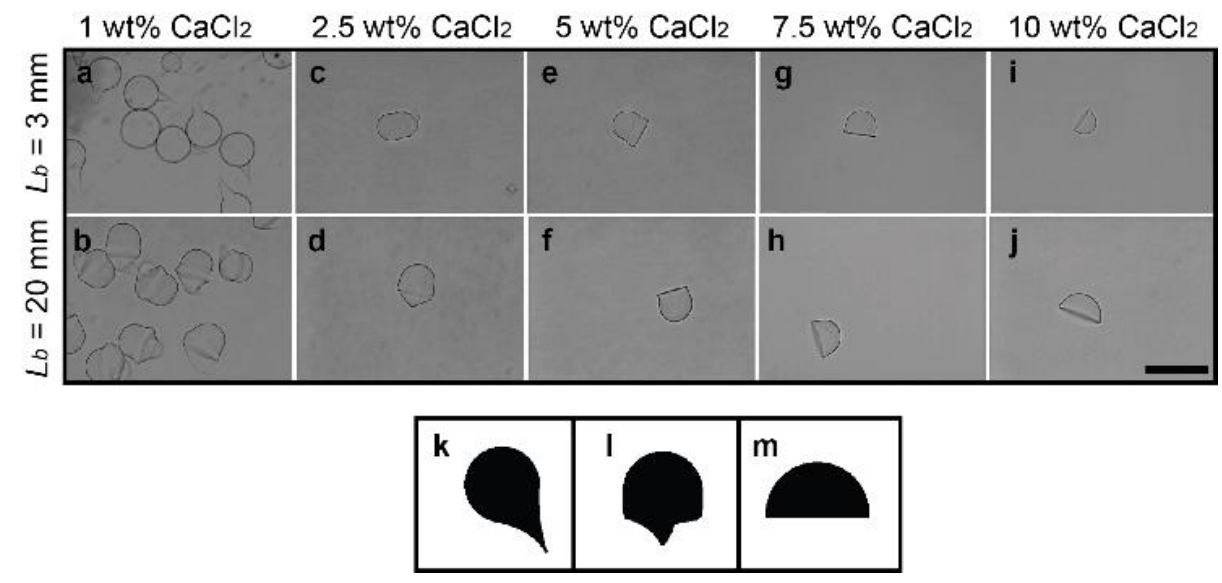

Figure 1 | Particle shape controlled by electrospray properties. a) Tadpole-like microparticles are generated when the ring-tobath distance $L_{b}=3 \mathrm{~mm}$ and the calcium chloride concentration $C=1 \mathrm{wt} \%$. b-d) In all other cases in our experiments, where the calcium chloride concentration $C \leq 2.5$ wt $\%$, we obtain cupcake-like particles. e-j) Hemispherical shaped particles are produced when the calcium chloride concentration $C \geq 5 \mathrm{wt} \%$. In all of these experiments, the voltage of the electrospray, and the flow rate of the pump are fixed at $5 \mathrm{kV}$ and $0.1 \mathrm{~mL} / \mathrm{hr}$, respectively. Scale bar represents $150 \mu \mathrm{m}$. Illustrations that more clearly show what we define as k) tadpole-like, I) cupcake-like, and $\mathbf{m}$ ) hemispherical shape microparticles.

The morphology of microparticles is important in controlling the efficacy of drug delivery and 3D cell culturing. ${ }^{[8]}$ Figure 1a-1j shows microscopic images of microparticles formed using our electrospray setup, with corresponding values of the ring-to-bath distance, $L_{b}$, and liquid bath calcium chloride concentration, C. We observe tadpole-like (Figure 1k), cupcake-like (Figure 1I), and hemispherical shape (Figure 1m) 
microparticles, depending on our experimental values of the ring-to-bath distance, $L_{b}$, and liquid bath calcium chloride concentration, $C$.

Notably, the tadpole-like and cupcake-like microparticles only form when the calcium chloride concentration $C<5$ wt\%. Tadpole-like shape particles occur when the ring-to-bath distance $L_{b}$ is low. When $L_{b}$ is high, cupcake-like particles form.

\subsection{Controlling the aspect ratio of hemispherical microparticles}

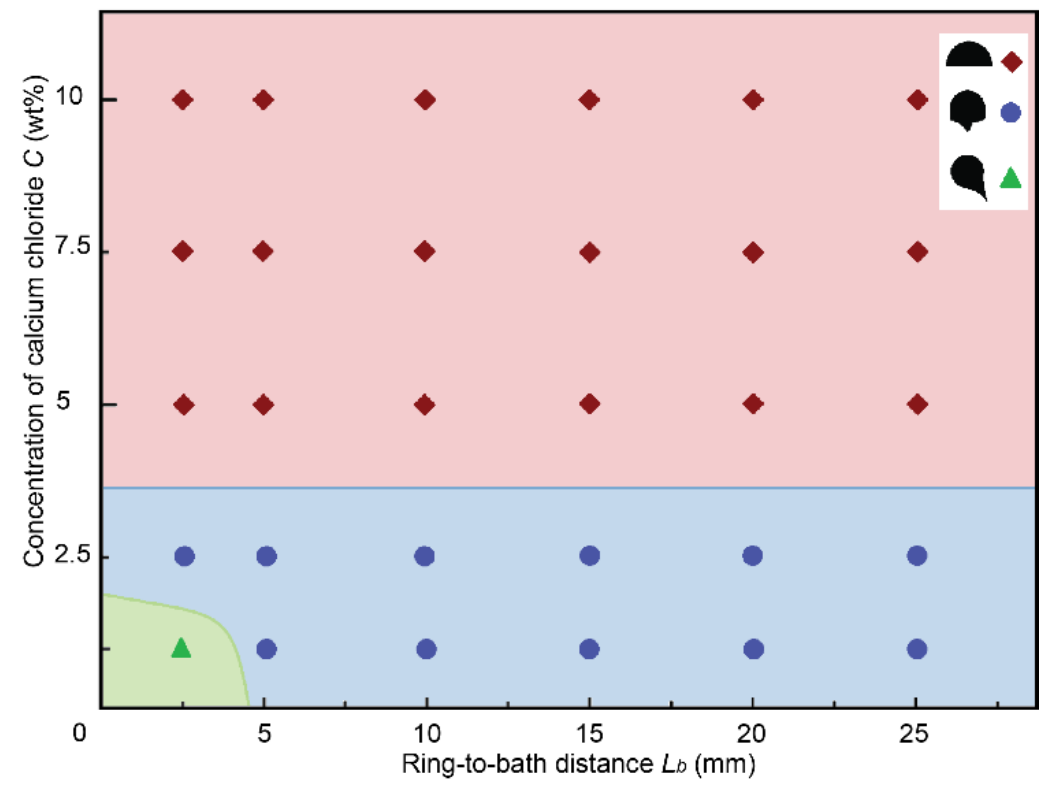

Figure 2 | Different types of alginate microparticles. A phase diagram of the generated calcium-alginate particles illustrates differences in particle shape as a result of changing the calcium chloride concentration $C$, and the ring-to-bath distance $L_{b}$. We find that the electrospray setup generates hemispherical particles as long as calcium chloride concentrations $C \geq 5$ wt\%. When calcium chloride concentration $C<5$ wt $\%$, we observe tadpole-like particles or cupcake-like particles.

The phase diagram in Figure 2 shows that when the liquid bath calcium chloride concentration $C \geq 5$ wt $\%$, our system produces only hemispherical microparticles. Our hypothesis is that, increasing the chemical initiator concentration, $C$, of the liquid bath, increases the alginate-calcium gelation rate. Therefore, when the alginate droplets from the electrospray impact the free surface of the bath, they deform such that the bottom side of the droplets flatten, and the immediate gelation of the alginate droplets "freezes" the droplets' hemispherical shapes (Figure 1e - 1j). (Further details of our hypothesis on the formation mechanism of hemispherical particles is discussed in Supporting Information, and shown in Figure S1.) In this regime, we define the particle aspect ratio $\Omega=h / b$, where $h$ and $b$ are the height and base length of the hemisphere, respectively (Figure 3a). In order to distinguish between different microparticle shape regimes, we define a Shape Index $S I=h-d / h+d$, where $d$ is the effective diameter of the microparticle. When $S I<0$, the fabricated microparticles are defined to be of hemispherical shape. For $S I>0$, we observe both tadpole-like and cupcake-like particles. To differenciate between tadpole-like and cupcake-like particles, we further define a 
Normalized Tail-Length $T L=w / d$, where $w$ is the width of the tail. When $T L<0.5$, tadpole-like microparticles are formed, and when $T L>0.5$, cupcake-like microparticles are produced (Further details of how the shapes are defined are discussed in Supporting Information).

Figure $3 b$ shows that increasing the calcium chloride concentration $C$ results in decreasing aspect ratio $\Omega$. There is also a nearly monotonic decrease of the aspect ratio $\Omega$ with increasing ring-to-bath distance $L_{b}$. The decrease in aspect ratio $\Omega$ indicates that the hemispheres are flatter. Larger ring-to-bath distances $L_{b}$ also result in more repeatable control of particle shape, as indicated by the aspect ratio $\Omega$ 's smaller error bars.

a
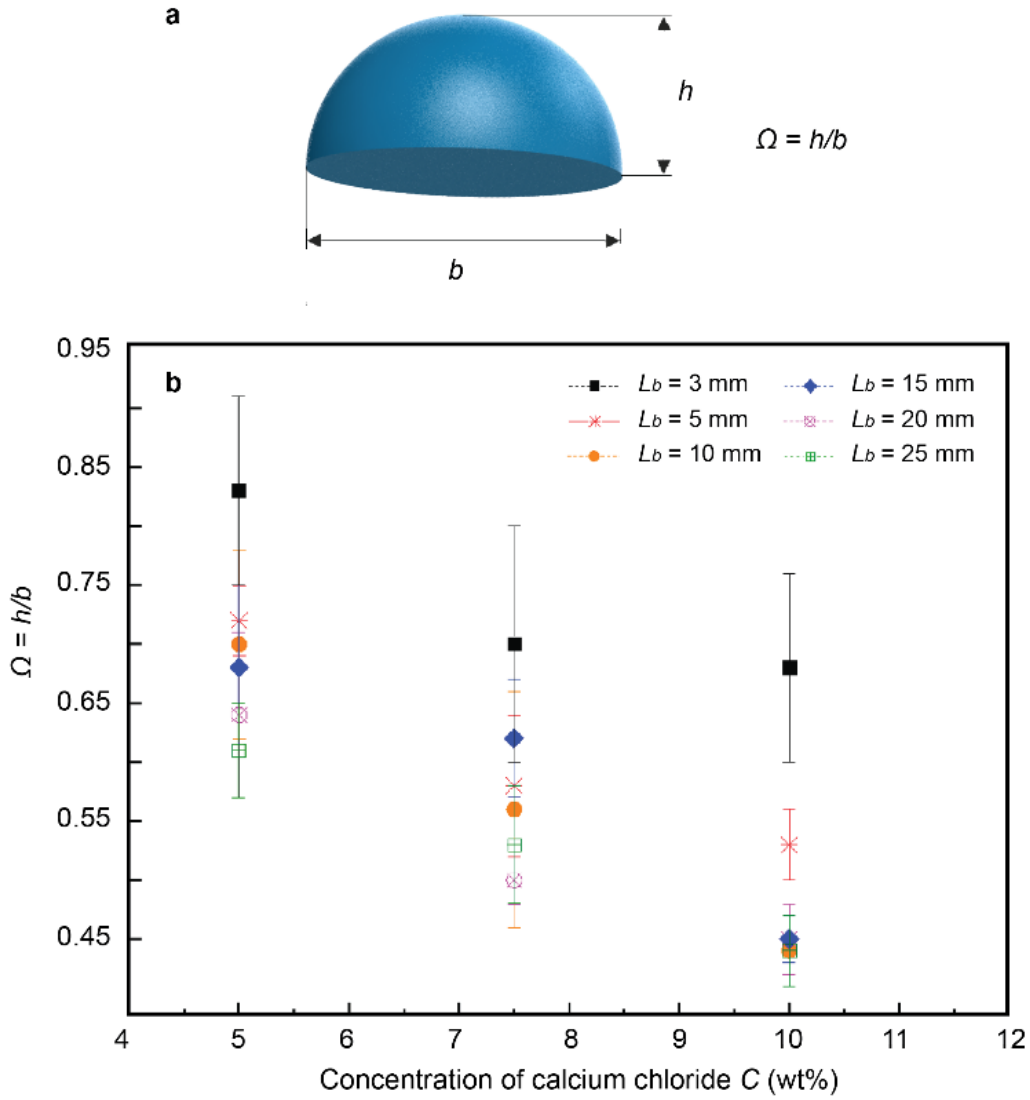

Figure 3 | Hemispherical particle aspect ratio $\Omega=h / b$ controlled by the liquid bath calcium chloride concentration $C$ and the ring-to-bath distance $L_{b}$. a) $\Omega$ is defined as the ratio between the height $h$ of the hemisphere and its base length $b$. b) A plot of the resulting hemispherical particle aspect ratio $\Omega$ versus the calcium chloride concentration $C$. Different symbols represents distinct values of the ring-to-bath distance $L_{b}$. Increasing the ring-to-bath distance $L_{b}$ for a particular calcium chloride concentration $C$ results in a nearly monotonic decrease in particle aspect ratio $\Omega$. Increasing the calcium chloride concentration $C$, while fixing the ring-to-bath distance $L_{b}$ also results in a nearly monotonic decrease in $\Omega$. Error bars indicate one standard deviation in a sample size of 20 particles.

\subsection{Generating microparticles with curved tails}


An additional parameter that we can adjust in our electrospray setup is the flow of the liquid in the calcium chloride bath. In normal conditions, the liquid bath is static, however, we can simply use a magnetic stirrer to controllably create a flow in the liquid bath. As a proof-of-concept demonstration, we conduct an experiment using a ring-to-bath distance $L_{b}=10 \mathrm{~mm}$, and a calcium chloride concentration $C=10 \mathrm{wt} \%$, while stirring the bath. We generate different degrees of flow in the liquid bath by tuning the rotational rate of the magnetic stirrer in the bath at 50,250 , and $500 \mathrm{rpm}$.

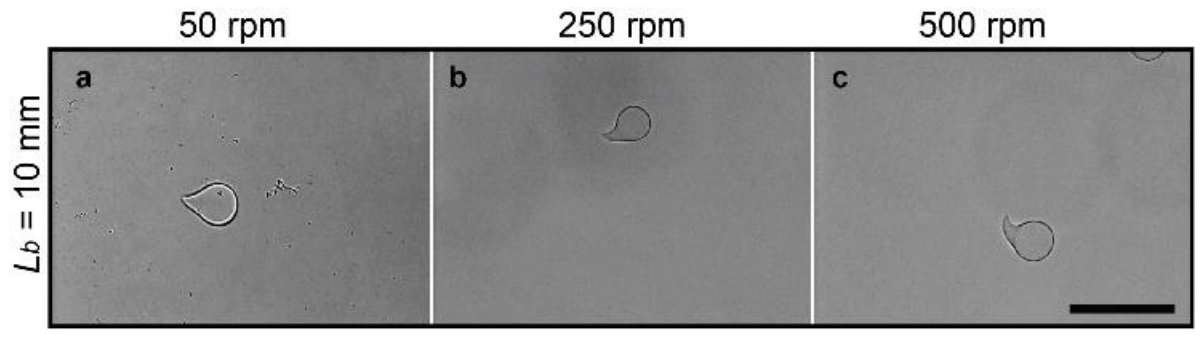

Figure 4 | The effect of stirring the liquid bath. We use a magnetic stirrer to create a flow in the liquid bath. Here, the ring-to-bath distance $L_{b}=10 \mathrm{~mm}$, and calcium chloride concentration $C=10 \mathrm{wt} \%$. As the magnetic stirrer rotation rate increases from a) 50 , b) 250 , to c) $500 \mathrm{rpm}$, the curvature of the resulting particle increases. Scale bar represents $150 \mu \mathrm{m}$.

Interestingly, we observe that the liquid bath flow results in the production of tear-shaped particles (Figure 4). When we increase the fluid flow by increasing the magnetic stirrer's rotational rate, we observe qualitatively, that the microparticle tails' curvature increases. We hypothesize that this curvature is influenced by the magnitude of shear stresses exerted on the microparticle, by the flow in the bath.

\subsection{Generating non-spherical magnetizable microparticles}

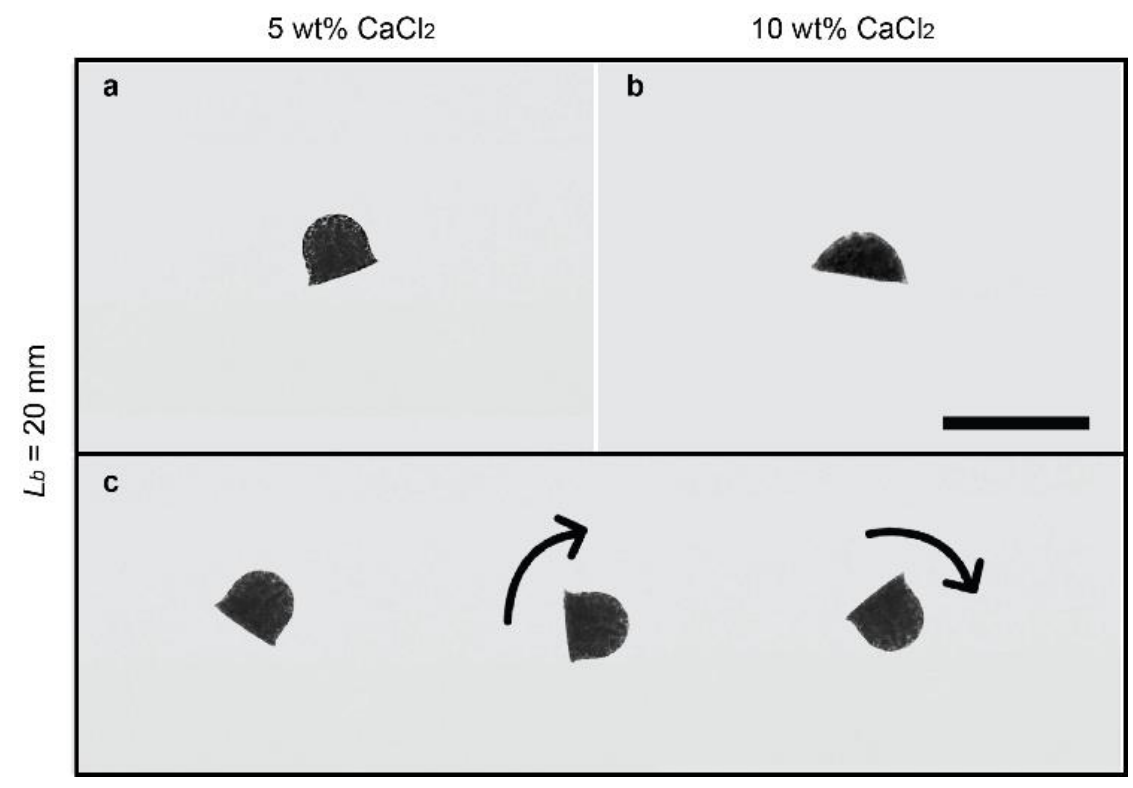

Figure 5 | Magnetic particles. Fabrication of magnetic microparticles using magnetic beads. The ring-to-bath distance is fixed at $L_{b}$ $=20 \mathrm{~mm}$, and two different calcium chloride concentrations a) $C=5$ and b) $C=10 \mathrm{wt} \%$ are used, resulting in different particle aspect 
ratios $\Omega$. c) A sequence of images showing that an external magnetic field from a moving permanent magnet causes a particle to rotate. Scale bar represents $150 \mu \mathrm{m}$.

Our technique can also be adapted to create microparticles that have the added functionality of magnetizability. Figure 5 shows magnetized non-spherical microparticles fabricated using our electrospray technique. Here, we add carboxylate-modified magnetic beads to the alginate precursor solution. As a proof-of-concept, we perform two sets of experiments with calcium chloride concentrations $C=5$ and 10 wt $\%$, and a ring-to-bath distance $L_{b}=20 \mathrm{~mm}$, while keeping all other parameters constant.

The fabricated magnetic microparticles are able to preserve the non-spherical shape of microparticles without magnetic function (Figure 5). These particles are magnetizable, and rotate in the presence of a moving magnetic field (see Figure 5 and also Supporting Information, Movie 1). This electrospray technique is therefore applicable to manufacturing magnetizable non-spherical particles at high throughput, for magnetic separation schemes. ${ }^{[36]}$

\subsection{Cell encapsulation}

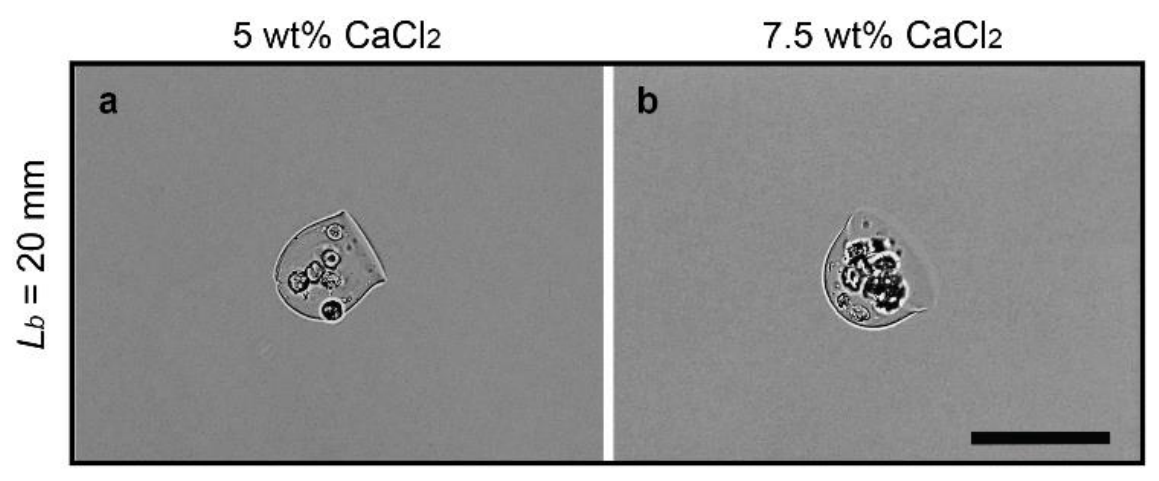

Figure 6 | Cell encapsulation. BT-474 breast cancer cells encapsulated in hemispherical microparticles using our electrospray technique. Here, the ring-to-bath distance is fixed at $L_{b}=20 \mathrm{~mm}$, and we use two different calcium chloride concentrations, a) $C=5$ and b) $C=7.5 \mathrm{wt} \%$. Scale bar represents $150 \mu \mathrm{m}$.

Figure 6 illustrates the encapsulation of breast cancer cells in non-spherical microparticles. The suspension of cells in alginate is sprayed into the bath, using our electrospray technique, and upon gelation of the particle, cells are encapsulated. Cell encapsulation in non-spherical microparticles, such as the ones shown here, may be desirable for immunoisolation of cells. ${ }^{[37-39]}$ This platform has the potential to achieve mass production of encapsulated cells, while providing control on the size and the shape of the cell encapsulating vehicles.

\section{Conclusions}

Even though microparticle shape is an important parameter in determining the effectiveness of drug delivery and 3D cell culturing, there are very few established methods to generate non-spherical biocompatible 
microparticles in a high throughput manner. The electrospray-based technique for producing non-spherical alginate microparticles, which we describe, provides precise control over the shape of biocompatible microparticles produced, by simply tuning the experimental parameters of the system.

We demonstrate that the setup's ring-to-bath distance $L_{b}$ and the calcium chloride concentration $C$ can both be used to control the final shape of the microparticles. We show the proof-of-concept production of particles that have tunable curvature tails, by magnetically stirring the liquid calcium chloride bath. We also show that the technique can be applied to generate magnetizable and cell encapsulating non-spherical alginate microparticles. Since electrospray is a method that generates particles at high throughput, ${ }^{[35,40]}$ we anticipate that this new non-spherical particle generation method will find utility in biomedical applications that benefit from shape-controlled biocompatible microparticles, such as cell encapsulation, drug delivery, and $3 \mathrm{D}$ cell culturing.

\section{Experimental Section}

\subsection{Chemicals and cells}

We prepare the alginate droplet phase solution as $2 \mathrm{wt} \%$ alginic acid sodium salt (Sigma-Aldrich Corp., St. Louis, MO, USA) in deionized (DI) water. The liquid bath phase is made with either $1 \mathrm{wt} \%, 2.5 \mathrm{wt} \%, 5 \mathrm{wt} \%$, $7.5 \mathrm{wt} \%$, or $10 \mathrm{wt} \%$ calcium chloride dihydrate (Sigma-Aldrich Corp., St. Louis, MO, USA) in DI water. We pass the alginate solution through a syringe filter that has $0.45 \mu \mathrm{m}$ size pores (Corning Inc., NY, USA) prior to use, to remove particulates and impurities from the droplet phase. We use $0.945 \mu \mathrm{m}$ diameter Sera-Mag carboxylate-modified magnetic speed-beads (Thermo Scientific, Waltham, MA, USA) in experiments that generate magnetizable non-spherical microparticles. We centrifuge $2 \mathrm{ml}$ of the magnetic bead solution until form a cluster at the bottom. Then, we remove the liquid content of the magnetic bead solution, add the alginate solution, and suspend the magnetic beads by mixing the solution. We prepare the magnetic alginate solution this way so that the alginate concentration is unaltered upon addition of the magnetic beads.

In cell encapsulation experiments, we use BT-474 breast cancer cells (ATCC, Manassas, VA, USA), cultured using Dulbecco's modified eagle's medium (DMEM) with 10\% fetal bovine serum (FBS). We incubate the cells at $37{ }^{\circ} \mathrm{C}$ with $5 \% \mathrm{CO}_{2}$ in a T-25 flask. We measure the concentration of cells by transferring $10 \mu \mathrm{L}$ of the cell sample to a hemocytometer (Hausser Scientific, Horsham, PA, USA). The calculated number of cells is approximately $2.8 \times 10^{6}$ cells per $\mathrm{mL}$. We centrifuge the cells in to remove the growth medium, and subsequently, we wash the cells with PBS to remove all the remaining DMEM. Then, we transfer the cells to a $1 \mathrm{~mL}$ alginate solution.

\subsection{Experimental setup}




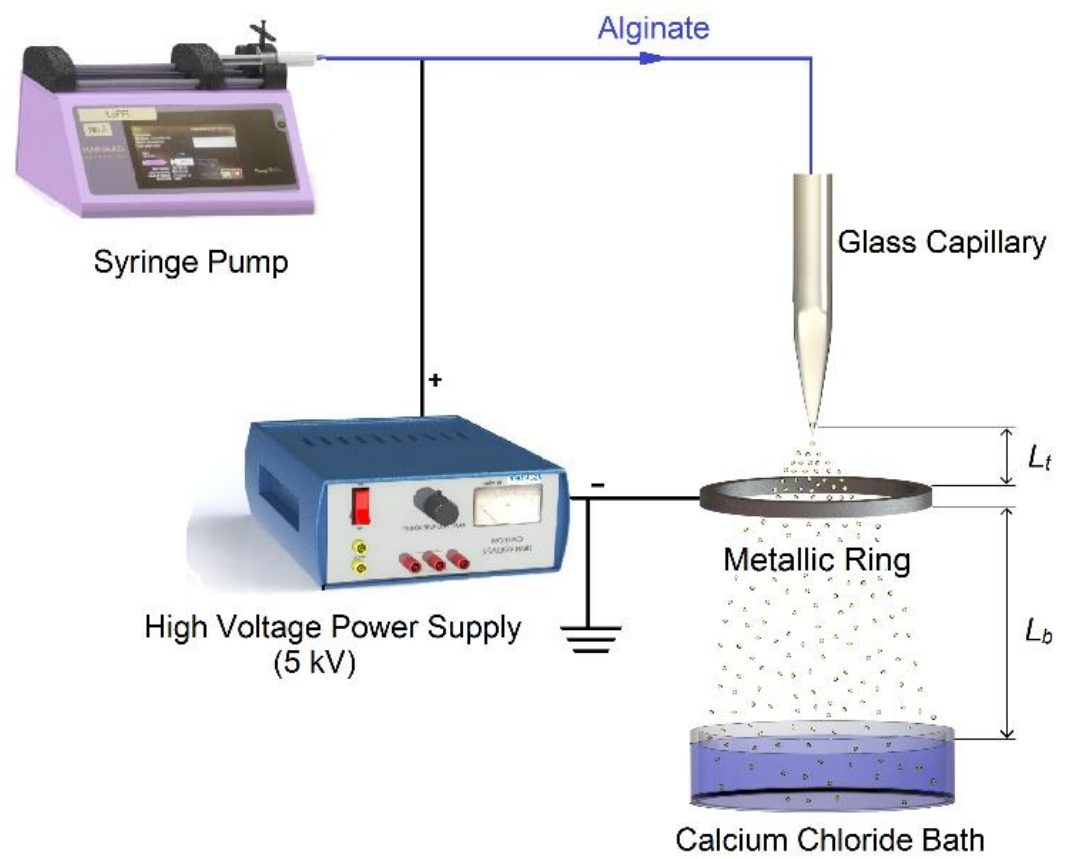

Scheme 1 | Schematic diagram of the electrospray system for alginate particle generation. A high-voltage power supply with low current is used to generate the electric field. The positively charged droplet phase is electrosprayed into a calcium chloride bath through a tapered glass capillary, to generate particles. The distance between the tip of the glass capillary and the ring is fixed at 1 $\mathrm{mm}$. We adjust the distance between the ring and the free-surface of the calcium chloride bath, $L_{b}$, to observe its effects on the resulting particle shapes.

Scheme 1 shows a schematic diagram of the experimental setup. We use a high-voltage power supply (Pasco Scientific, Roseville, CA, USA), with a constant voltage of $5 \mathrm{kV}$, to generate an electric field. The power supply is connected to a metallic needle on the dispensing syringe through a wire to positively charge the droplet phase. A metallic ring is also connected to the power supply, which is negatively charged. We use a constant flow rate syringe pump (Harvard Apparatus, Holliston, MA, USA), set at a flow rate of 0.1 $\mathrm{mL} / \mathrm{hr}$, to pump the droplet phase through tubing (Saint-Gobain Corp., Malvern, PA, USA) to a borosilicate glass capillary ( $1 \mathrm{~mm}$ O.D. and $0.25 \mathrm{~mm}$ I.D.; A-M System Inc., Sequim, WA, USA). The tip of the glass capillary is tapered, using a micropipette puller (Model P-97, Sutter Instruments, Novato, CA, USA), so that the tip has a diameter of $50 \mu \mathrm{m}( \pm 5 \mu \mathrm{m})$. The internal and external surfaces of the glass capillary are coated with triethoxy(octyl)silane (Sigma-Aldrich Corp., St. Louis, MO, USA) to make the surfaces hydrophobic, which prevents wetting of the glass capillary and achieves smoother and more monodisperse dispensing.

Dispensed droplets pass through the negatively charged metallic ring under the effect of the electric field. As Scheme 1 shows, the distance between the glass capillary tip and the metallic ring, $L_{t}=1 \mathrm{~mm}$. In our experiments, we investigate the effects on the formation and morphology of the particles by adjusting two variables: the liquid bath calcium chloride concentration, $(C)$, and the metallic ring to liquid bath surface distance, $\left(L_{b}\right)$ (Scheme 1). After the particles are formed, we image the morphology and structure of the particles using an inverted Zeiss optical microscope (Carl Zeiss, Oberkochen, Germany). 


\section{Acknowledgements}

The authors thank S Buryk for an early review of this manuscript. SSH Tsai and DK Hwang are both grateful for funding from the Discovery grants program of the Canadian Natural Sciences and Engineering Research Council (NSERC). SY Mak and HC Shum both acknowledge funding support from the Research Grants Council of Hong Kong through the General Research Fund (Nos. HKU 719813E and 17304514). SY Mak thanks the Department of Mechanical Engineering in the University of Hong Kong for offering the Overseas Visiting Scheme to establish this collaborative research.

\section{References:}

[1] M. Das, H. Zhang, E. Kumacheva, Annu. Rev. Mater. Res. 2006, 36, 117-142.

[2] P. Panda, S. Ali, E. Lo, B. G. Chung, T. A. Hatton, A. Khademhosseini, P. S. Doyle, Lab Chip 2008, 8, 1056.

[3] L. K. Fiddes, E. W. K. Young, E. Kumacheva, A. R. Wheeler, Lab Chip 2007, 7, 863-7.

[4] H. Zhang, E. Tumarkin, R. M. A. Sullan, G. C. Walker, E. Kumacheva, Macromol. Rapid Commun. 2007, 28, 527-538.

[5] J. A. Champion, Y. K. Katare, S. Mitragotri, J. Control. Release 2007, 121, 3-9.

[6] O. Pillai, R. Panchagnula, Curr. Opin. Chem. Biol. 2001, 5, 447-451.

[7] S. Martins, B. Sarmento, E. B. Souto, D. C. Ferreira, Carbohydr. Polym. 2007, 69, 725-731.

[8] E. A. Simone, T. D. Dziubla, V. R. Muzykantov, Expert Opin. Drug Deliv. 2008, 5, 1283-1300.

[9] J. Panyam, M. M. Dali, S. K. Sahoo, W. Ma, S. S. Chakravarthi, G. L. Amidon, R. J. Levy, V. Labhasetwar, J. Control. Release 2003, 92, 173-187.

[10] J. Sun, H. Tan, Materials (Basel). 2013, 6, 1285-1309.

[11] S. Utech, R. Prodanovic, A. S. Mao, R. Ostafe, D. J. Mooney, D. a. Weitz, Adv. Healthc. Mater. 2015, 4, $1628-1633$.

[12] A. G. Håti, D. C. Bassett, J. M. Ribe, P. Sikorski, D. A. Weitz, B. T. Stokke, Lab Chip 2016, 16, 3718-3727.

[13] W. H. Tan, S. Takeuchi, Adv. Mater. 2007, 19, 2696-2701.

[14] S. Hua, H. Yang, Q. Li, J. Zhang, A. Wang, Drug Dev. Ind. Pharm. 2012, 38, 728-34.

[15] T. Andersen, B. L. Strand, K. Formo, E. Alsberg, B. E. Christensen, Carbohydr. Chem. Chem. Biol. Approaches, Vol. 37 2012, 227-258.

[16] Y. Tabata, S. Gutta, R. Langer, Pharm. Res. An Off. J. Am. Assoc. Pharm. Sci. 1993, 10, 487-496.

[17] M. Dunne, O. I. Corrigan, Z. Ramtoola, Biomaterials 2000, 21, 1659-1668.

[18] C. Berkland, K. Kim, D. W. Pack, J. Control. Release 2001, 73, 59-74.

[19] D. S. T. Hsieh, W. D. Rhine, R. Langer, J. Pharm. Sci. 1983, 72, 17-22.

[20] E. A. Simone, T. D. Dziubla, D. E. Discher, V. R. Muzykantov, Biomacromolecules 2009, 10, 1324-1330.

[21] Y. Geng, P. Dalhaimer, S. Cai, R. Tsai, M. Tewari, T. Minko, D. E. Discher, Nat Nano 2007, 2, $249-255$.

[22] E. S. Chan, B. B. Lee, P. Ravindra, D. Poncelet, J. Colloid Interface Sci. 2009, 338, 63-72.

[23] F. Davarci, D. Turan, B. Ozcelik, D. Poncelet, Food Hydrocoll. 2017, 62, 119-127.

[24] D. An, A. Warning, K. G. Yancey, C.-T. Chang, V. R. Kern, A. K. Datta, P. H. Steen, D. Luo, M. Ma, Nat. Commun. 2016, 7, 12401.

[25] S. Xu, Z. Nie, M. Seo, P. Lewis, E. Kumacheva, H. A. Stone, P. Garstecki, D. B. Weibel, I. Gitlin, G. M. Whitesides, Angew. 
Chemie - Int. Ed. 2005, 44, 724-728.

[26] D. Dendukuri, K. Tsoi, T. A. Hatton, P. S. Doyle, Langmuir 2005, 21, 2113-2116.

[27] D. Dendukuri, D. C. Pregibon, J. Collins, T. A. Hatton, P. S. Doyle, Nat. Mater. 2006, 5, 365-369.

[28] J. P. Rolland, B. W. Maynor, L. E. Euliss, A. E. Exner, G. M. Denison, J. M. DeSimone, J. Am. Chem. Soc. 2005, 127, 1009610100.

[29] T. S. Shim, S.-M. Yang, S.-H. Kim, Nat. Commun. 2015, 6, 6584.

[30] D. K. Hwang, J. Oakey, M. Toner, J. A. Arthur, K. S. Anseth, S. Lee, A. Zeiger, K. J. Van Vliet, P. S. Doyle, J. Am. Chem. Soc. 2009, 131, 4499-4504.

[31] D. K. Hwang, D. Dendukuri, P. S. Doyle, Lab Chip 2008, 8, 1640-1647.

[32] O. Jeon, K. H. Bouhadir, J. M. Mansour, E. Alsberg, Biomaterials 2009, 30, 2724-2734.

[33] Q. Gao, Y. He, J. zhong Fu, J. jiang Qiu, Y. an Jin, J. Sol-Gel Sci. Technol. 2016, 77, 610-619.

[34] Y. Hu, Q. Wang, J. Wang, J. Zhu, H. Wang, Y. Yang, Biomicrofluidics 2012, 6, 1-9.

[35] C. D. Hendricks, S. Babil, J. Phys. E. 1972, 5, 905-910.

[36] R. Zhou, F. Bai, C. Wang, Lab Chip 2017, 17, 401-406.

[37] G. Orive, R. M. Hernández, A. Rodríguez Gascón, R. Calafiore, T. M. S. Chang, P. De Vos, G. Hortelano, D. Hunkeler, I. Lacík, J. L. Pedraz, Trends Biotechnol. 2004, 22, 87-92.

[38] R. H. Li, Adv. Drug Deliv. Rev. 1998, 33, 87-109.

[39] L. M. Weber, J. He, B. Bradley, K. Haskins, K. S. Anseth, Acta Biomater. 2006, 2, 1-8.

[40] S. M. Naqvi, S. Vedicherla, J. Gansau, T. McIntyre, M. Doherty, C. T. Buckley, Adv. Mater. 2016, 5662-5671. 